\title{
Evaluation of retinal and choroidal variations in thyroid-associated ophthalmopathy using optical coherence tomography angiography
}

Lanchu Yu ${ }^{1+}$, Qin Jiao ${ }^{1+}$, Yu Cheng ${ }^{1}$, Yanji Zhu' ${ }^{1}$ Zhongjing Lin $^{2^{*}}$ (i) and Xi Shen ${ }^{1 *}$

\begin{abstract}
Background: To investigate the difference in retinal nerve fiber layer (RNFL) thickness, choroidal thickness (CT) and superficial retinal vessels between thyroid-associated ophthalmopathy (TAO) patients and healthy controls. To identify the potential influencing factors for these parameters and evaluate their diagnostic abilities in TAO.

Methods: Twenty active TAO patients, 33 inactive TAO patients and 29 healthy participants were enrolled. TAO patients were divided according to the clinical activity score (CAS). RNFL thickness and CT were measured by HDOCT, while foveal avascular zone (FAZ), vascular density and perfusion density were measured by optical coherence tomography angiography (OCTA). SPSS software was used for statistical analysis.

Results: Active TAO patients had thinner RNFL thickness than the other two groups $(P<0.001, P<0.001)$. Both active and inactive TAO patients had significantly higher $C T$ in the macular region (all $P<0.05$ ). The FAZ area in the active TAO group was significantly larger than the other two groups $(P=0.045, P=0.001)$. The inactive TAO group had significantly higher vascular density than the other two groups (all $P<0.05$ ). With regard to the perfusion density, significant differences were observed in the temporal and inferior areas $(P=0.045, P=0.001)$, as well as the average values $(P=0.032)$. The FAZ area was positively correlated with intraocular pressure $(r=0.274, P=0.013)$, while it was negatively correlated with axial length $(r=-0.344, P=0.002)$. The vascular density and perfusion density were not significantly correlated with different clinical variables (all $P>0.05$ ). The AUC analysis indicated these parameters also exhibited a significant discriminatory power in TAO diagnosis.

Conclusions: TAO patients had significant variations in RNFL thickness, choroidal thickness, FAZ area and superficial retinal vessels. These parameters appeared to be potential adjuncts in the evaluation of TAO patients.
\end{abstract}

Keywords: Thyroid-associated ophthalmopathy, Optical coherence tomography angiography, Choroidal thickness, Retinal nerve fiber layer

\footnotetext{
*Correspondence: ahwwlzj@126.com; carl_shen2005@126.com

${ }^{\dagger}$ Lanchu Yu and Qin Jiao contributed equally to this work.

2Department of Ophthalmology, Ren Ji Hospital, School of Medicine, Renji Hospital Affiliated Medical School, Shanghai Jiao Tong University, 160 Pu Jian Road, Shanghai 200127, China

${ }^{1}$ Department of Ophthalmology, Rui Jin Hospital, School of Medicine,

Shanghai Jiao Tong University, 197 Rui Jin Er Road, Shanghai 200025, China
}

(c) The Author(s). 2020 Open Access This article is licensed under a Creative Commons Attribution 4.0 International License, which permits use, sharing, adaptation, distribution and reproduction in any medium or format, as long as you give appropriate credit to the original author(s) and the source, provide a link to the Creative Commons licence, and indicate if changes were made. The images or other third party material in this article are included in the article's Creative Commons licence, unless indicated otherwise in a credit line to the material. If material is not included in the article's Creative Commons licence and your intended use is not permitted by statutory regulation or exceeds the permitted use, you will need to obtain permission directly from the copyright holder. To view a copy of this licence, visit http://creativecommons.org/licenses/by/4.0/. The Creative Commons Public Domain Dedication waiver (http://creativecommons.org/publicdomain/zero/1.0/) applies to the data made available in this article, unless otherwise stated in a credit line to the data. 


\section{Background}

Thyroid-associated ophthalmopathy (TAO) is a systemic autoimmune disorder or an organ-specific autoimmune inflammatory disease of orbital tissues. It is characterized by inflammatory cellular infiltration with lymphocytes, plasma cells, macrophages and mast cells. The most obvious pathological changes in orbital tissues include interstitial tissue edema, orbital fat hyperplasia and massively swollen extraocular muscles, which may cause orbital compression symptoms [1-4]. Approximately $20 \%$ of patients with immune thyroid diseases will develop TAO, and $25-50 \%$ of TAO cases are closely related to hyperthyroidism, commonly termed as Graves' ophthalmopathy, with higher morbidity in females [3, 4]. The exact pathogenesis of TAO remains unknown, but the clinical manifestations can be explained by the expansion of orbital volume due to autoimmune inflammatory infiltration. TAO can be classified into the following phases: an active phase with rapid progression and an inactive phase with symptom stabilization $[5,6]$. However, approximately $3-5 \%$ of TAO patients in an inactive state will transit to an active state, which may result in aggravation of proptosis, lid retraction, dysfunctional eye motility, or even vision loss due to optic nerve compression [7, 8].

Different techniques have been employed to analyze the retinal and choroidal changes in TAO patients. Walasik-Szemplińska et al. [9] observed the alterations of ocular blood supply in TAO patients by color Doppler imaging. However, there are several influencing factors in the measuring process of ocular blood flow, such as eyeball movement and Doppler angles. The diameters of the examined vessels also limit its clinical applications. Optical coherence tomography (OCT) is a non-invasive imaging technique that used to visualize the detailed structures of the retina and choroid. Sayin et al. [10] found TAO patients had thinner inferior retinal nerve fiber layer (RNFL) thickness and macular thickness. Furthermore, choroidal thickness (CT) was found to be increased in TAO patients $[11,12]$. With the advancement in technology, OCT angiography (OCTA) is a new promising imaging technique that is capable of imaging the retinal and choroidal vasculature noninvasively [1315]. Recently, OCTA has been widely used in ophthalmic diseases such as glaucoma and diabetic eye disease. However, few studies have investigated the retinal vessels in the macular region in TAO patients using OCTA.

Currently, the evaluation of TAO has to be done qualitatively according to the clinical activity score (CAS) system. If the evaluation can be carried out quantitatively, the disease severity will be determined with high accuracy. Therefore, we aimed to examine the detailed retinal and choroidal variations in patients with active and inactive TAO using OCTA. We also aimed to explore their correlations with different ocular parameters and investigate their clinical diagnostic capability when comparing with healthy controls.

\section{Methods \\ Study population}

In our study, all subjects were recruited from the Department of Ophthalmology at Ruijin Hospital, Shanghai Jiao Tong University School of Medicine between December 2015 and December 2017. All participants signed informed consent forms to participate in the study and received immediate medical attention when needed.

TAO patients were divided into two groups according to the CAS [16]: (1) spontaneous orbital pain, (2) gazeevoked orbital pain, (3) eyelid swelling, (4) eyelid erythema, (5) conjunctival redness, (6) chemosis, and (7) caruncle inflammation or plica. Patients with $\mathrm{CAS} \geq 3 / 7$ were classified as active TAO, and those with CAS $\leq 2 / 7$ were classified as inactive TAO. All TAO patients were newly diagnosed. Age-matched healthy volunteers seeking physical examinations were enrolled as the control group in the same time period. The exclusion criteria for both patients and healthy participants were as follows: (1) special treatment for thyroid diseases within 3 months, such as radioactive iodine therapy, immunosuppressor agents and thyroid surgery; (2) hormonotherapy within 6 months; (3) history of ocular surgical procedures; (4) concomitant ocular diseases, such as glaucoma, retinal vein occlusion, and maculopathy; (5) concurrent infection or severe systemic diseases.

All participants underwent a complete ophthalmic examination including best-corrected visual acuity, intraocular pressure (IOP) measurement with Goldmann applanation tonometry, slit lamp examination and fundus examination. B-scan ultrasonography was performed to assess the ocular and orbital structure. Central corneal thickness (CCT) and axial length (AL) were recorded using Lenstar LS900 (Haag-Streit AG, Switzerland). The proptosis was measured by the same examiner who was experienced at Hertel exophthalmometry.

\section{Image acquisition and processing}

All participants were examined with a traditional highdefinition OCT system (Carl Zeiss Meditec, Dublin, CA, USA). The optic disc cube $200 \times 200$ mode was conducted to obtain RNFL results. CT measurements were performed in the superior, inferior, nasal and temporal regions with selected locations, including $500 \mu \mathrm{m}$, $1000 \mu \mathrm{m}, 1500 \mu \mathrm{m}$, and $2000 \mu \mathrm{m}$ from the fovea. CT was defined as the distance between the hyper-reflective line of Bruch's membrane and the innermost hyper-reflective line of the choroidoscleral interface [17]. 
OCTA images of the macula were obtained using a Cirrus high-definition OCT prototype with AngioPlex (Carl Zeiss Meditec, Dublin, CA, USA). The macula was imaged using a $3 \times 3 \mathrm{~mm}$ scan pattern. Tracking technology was applied to reduce the effect of motion artifacts. Only high-quality images with signal strengths over 8 were included for analysis. Parameters to evaluate the superficial retinal vessels (from inner boundary membrane layer to the inner plexus layer), including foveal avascular zone (FAZ), vascular density and perfusion density, were calculated using the manufacturer's angiometric software (Fig. 1). Vascular density is the linear length of vessels divided by the selected area. Perfusion density represents the area of vessels distribution divided by the selected area. Although both eyes were eligible for our study, only right eye was selected in the final data analysis.

\section{Statistical analysis}

The Statistical Package for Social Sciences version 22.0 for Windows (SPSS Inc., IBM Corp., Chicago, IL, USA) was used for data analysis. The Levene test was selected to assure the variance homogeneity. The KolmogorovSmirnov test was performed to check the normality of the data distributions. Chi-square tests were used to analyze the categorical variables. For comparisons of normally distributed data among three groups, the oneway analysis of variance was chosen, and least square difference (LSD) test was subsequently performed for group comparisons; otherwise, a Kruskal-Wallis test was adopted, and then a Mann-Whitney $U$ test was used for group comparisons. Pearson's correlation coefficients were calculated to evaluate the relationship between different clinical parameters. The receiver operating characteristic (ROC) curve and the area under curve (AUC) were conducted to assess the diagnostic capability of different parameters in TAO. Sensitivities at fixed specificities (85 and 95\%) were calculated for different parameters. A $P$ value of $<0.05$ was considered statistically significant in our analysis.

\section{Results}

A total of 82 eligible subjects were enrolled, including 20 active TAO patients, 33 inactive TAO patients, and 29 healthy participants. The basic characteristics of all participants are shown in Table 1. There were no significant differences in age, sex distributions, AL and CCT among the three groups $(P=0.339, P=0.121, P=0.100, P=$ 0.633 , respectively). As expected, the proptosis in active TAO patients $(21 \pm 3)$ was highest, followed by inactive
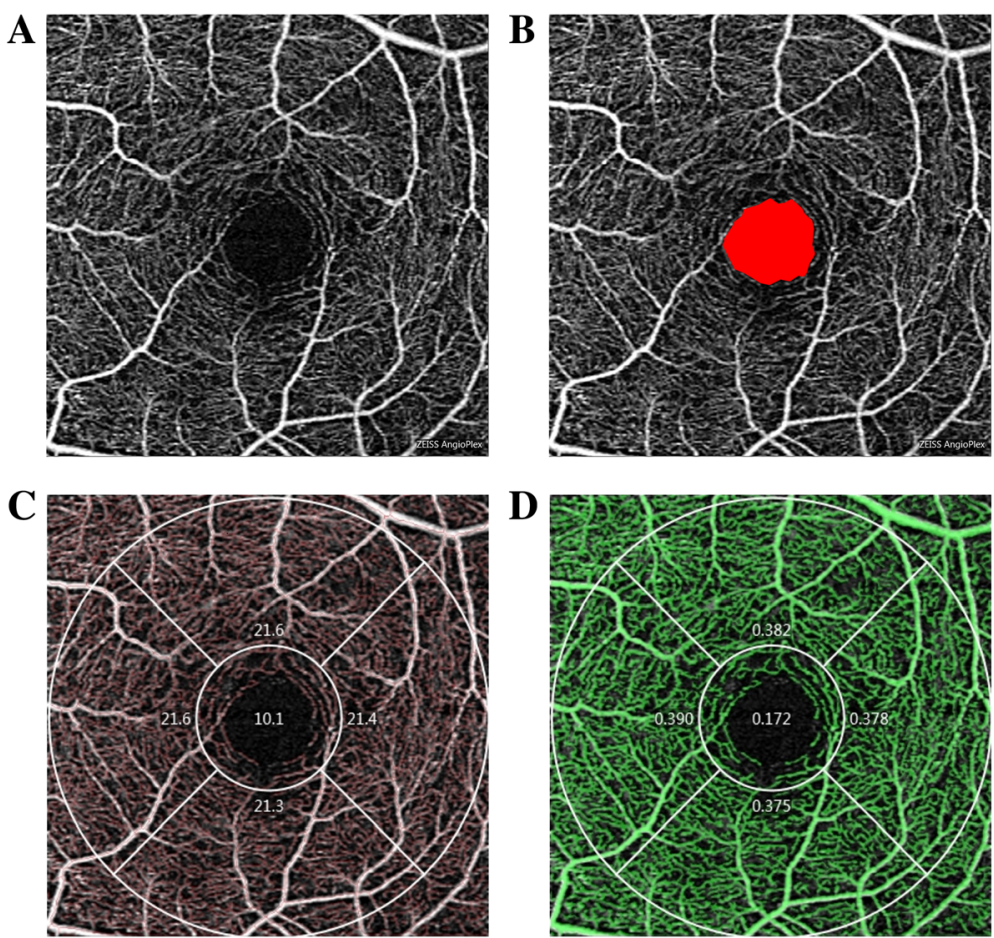

Fig. 1 The $3 \times 3 \mathrm{~mm}$ optical coherence tomography angiography image of the macular region of the retina (a) OCTA image showing the detailed structure of superficial retinal vessels (b) OCTA image showing the FAZ area (c) OCTA image showing the measurement of vascular density in the macular region (d) OCTA image showing the measurement of perfusion density in the macular region 
Table 1 The basic characteristics of the study population

\begin{tabular}{lllll}
\hline & Active TAO & Inactive TAO & Normal & $\boldsymbol{P}$ value \\
\hline Age (years) & $43.5 \pm 11.5$ & $39.3 \pm 11.3$ & $38.7 \pm 12.5$ & 0.339 \\
Sex (F/M) & $12 / 8$ & $27 / 6$ & $24 / 5$ & 0.121 \\
Proptosis $(\mathrm{mm})$ & $21 \pm 3$ & $18 \pm 3$ & $16 \pm 2$ & $<0.001$ \\
IOP $(\mathrm{mmHg})$ & $22.6 \pm 4.3$ & $16.5 \pm 3.3$ & $15.4 \pm 2.6$ & $<0.001$ \\
$\mathrm{AL}(\mathrm{mm})$ & $23.53 \pm 1.28$ & $24.08 \pm 1.01$ & $24.20 \pm 1.08$ & 0.100 \\
$\mathrm{CCT}(\mu \mathrm{m})$ & $541 \pm 27$ & $540 \pm 27$ & $534 \pm 30$ & 0.633 \\
\hline
\end{tabular}

TAO patients $(18 \pm 3)$ and healthy controls $(16 \pm 2)$ $(P<0.001)$. Active TAO patients also had higher IOP than the other two groups $(P<0.001)$.

The traditional OCT analysis results are summarized in Table 2. The global average RNFL thickness was significantly different among the three groups $(P<0.001)$, post hoc pairwise comparisons revealed that active TAO patients had thinner RNFL thickness than the other two groups $(P<0.001, P<0.001)$. Similar results were obtained when comparing the temporal and inferior RNFL thickness $(P=0.001, P=0.001)$, while no significant differences were observed in superior and nasal RNFL thickness $(P=0.458, P=0.117)$. With regard to $C T$ in the macular region, TAO patients, no matter active or inactive, both had significantly higher CT than healthy individuals (all $P<0.05$ ). However, no significant differences were detected between active and inactive TAO patients (all $P>0.05$ )

The evaluation of the superficial retinal vessels measured by OCTA are summarized in Table 3 . The mean area of FAZ in the active TAO group was $0.36 \pm 0.09$ $\mathrm{mm}^{2}$, which was significantly larger than the other two groups $(P=0.045, P=0.001)$. In contrast, there was no significant difference between the inactive TAO group and control group $(P=0.130)$. However, the inactive TAO group had significantly higher vascular density than the other two groups (all $P<0.05$ ), while there were no significant differences between the active TAO group and normal group (all $P>0.05$ ). With regard to the perfusion density, significant differences were observed in the temporal and inferior areas $(P=0.045, P=0.001)$, as well as the average values $(P=0.032)$. But the pairwise comparison results were not completely consistent.

To determine the potential influencing factors associated with these above parameters, Pearson's correlation coefficients were calculated with the clinical variables of all the 82 eyes (Table 4). The FAZ area was positively correlated with IOP $(\mathrm{r}=0.274, P=0.013)$, while it was negatively correlated with axial length $(r=-0.344, P=$ $0.002)$. The vascular density and perfusion density were not significantly correlated with different clinical variables (all $P>0.05$ ). Moreover, to determine whether these parameters can be used in TAO diagnosis, ROC curves were generated (Table 5). Table 6 showed sensitivities at fixed specificities and their cut-off values. The AUC analysis indicated that RNFL thickness had modest diagnostic power in active $\mathrm{TAO} /$ inactive $\mathrm{TAO}$ and active $\mathrm{TAO} /$ normal subgroups $(\mathrm{AUC}=0.804, P<0.001$; $\mathrm{AUC}=$ $0.818, P<0.001)$. Comparisons of CT yielded ROC curve areas of 0.814 for active $\mathrm{TAO} /$ normal and 0.828 for inactive TAO/normal $(P<0.001, P<0.001)$. In contrast, FAZ area only exhibited a significant discriminatory power in active $\mathrm{TAO} /$ normal comparison $(\mathrm{AUC}=$ $0.711, P=0.013)$. The vascular density and perfusion density also showed significant diagnostic ability in active $\mathrm{TAO} /$ inactive $\mathrm{TAO}$ and inactive $\mathrm{TAO} /$ normal

Table $\mathbf{2}$ OCT analysis results in different study groups ( $\mu \mathrm{m})$

\begin{tabular}{|c|c|c|c|c|c|c|c|}
\hline & Active TAO & Inactive TAO & Normal & $P$ value & P1 & P2 & P3 \\
\hline \multicolumn{8}{|c|}{ RNFL thickness } \\
\hline Average & $92 \pm 7$ & $101 \pm 8$ & $102 \pm 8$ & $<0.001$ & $<0.001$ & $<0.001$ & 0.999 \\
\hline Superior & $116 \pm 15$ & $121 \pm 18$ & $122 \pm 16$ & 0.458 & & & \\
\hline Temporal & $72 \pm 10$ & $84 \pm 13$ & $83 \pm 13$ & 0.001 & 0.002 & 0.004 & 0.991 \\
\hline Inferior & $117 \pm 11$ & $133 \pm 16$ & $132 \pm 15$ & 0.001 & 0.001 & 0.004 & 0.932 \\
\hline Nasal & $63 \pm 7$ & $68 \pm 10$ & $67 \pm 9$ & 0.117 & & & \\
\hline \multicolumn{8}{|c|}{ Choroidal thickness } \\
\hline Average & $257 \pm 37$ & $260 \pm 42$ & $218 \pm 32$ & $<0.001$ & 0.795 & $<0.001$ & $<0.001$ \\
\hline Subfoveal & $304 \pm 41$ & $299 \pm 45$ & $258 \pm 38$ & $<0.001$ & 0.676 & $<0.001$ & $<0.001$ \\
\hline Superior & $270 \pm 33$ & $274 \pm 42$ & $216 \pm 30$ & $<0.001$ & 0.753 & $<0.001$ & $<0.001$ \\
\hline Temporal & $261 \pm 42$ & $265 \pm 41$ & $223 \pm 38$ & $<0.001$ & 0.752 & 0.002 & $<0.001$ \\
\hline Inferior & $251 \pm 36$ & $264 \pm 41$ & $216 \pm 30$ & $<0.001$ & 0.193 & 0.001 & $<0.001$ \\
\hline Nasal & $235 \pm 50$ & $241 \pm 45$ & $203 \pm 38$ & 0.004 & 0.655 & 0.016 & 0.001 \\
\hline
\end{tabular}

P1: $P$ value for the comparison group between active TAO and inactive TAO 
Table 3 OCTA analysis results in different study groups

\begin{tabular}{|c|c|c|c|c|c|c|c|}
\hline & Active TAO & Inactive TAO & Normal & $P$ value & P1 & P2 & P3 \\
\hline FAZ area $\left(\mathrm{mm}^{2}\right)$ & $0.36 \pm 0.09$ & $0.31 \pm 0.08$ & $0.28 \pm 0.08$ & 0.006 & 0.045 & 0.001 & 0.130 \\
\hline \multicolumn{8}{|c|}{ Vascular density $\left(\mathrm{mm}^{-1}\right)$} \\
\hline Superior & $21.1 \pm 1.5$ & $22.0 \pm 1.1$ & $21.4 \pm 1.8$ & 0.063 & & & \\
\hline Temporal & $20.3 \pm 1.6$ & $21.5 \pm 1.1$ & $20.7 \pm 2.1$ & 0.026 & 0.012 & 0.485 & 0.044 \\
\hline Inferior & $21.0 \pm 1.4$ & $21.9 \pm 1.3$ & $20.6 \pm 1.8$ & 0.004 & 0.027 & 0.481 & 0.001 \\
\hline Nasal & $21.2 \pm 1.4$ & $22.0 \pm 1.2$ & $21.5 \pm 1.9$ & 0.148 & & & \\
\hline Average & $21.0 \pm 1.1$ & $21.9 \pm 0.9$ & $21.0 \pm 1.7$ & 0.005 & 0.003 & 0.691 & 0.014 \\
\hline \multicolumn{8}{|l|}{ Perfusion density } \\
\hline Superior & $0.380 \pm 0.029$ & $0.393 \pm 0.021$ & $0.381 \pm 0.031$ & 0.097 & & & \\
\hline Temporal & $0.367 \pm 0.030$ & $0.386 \pm 0.020$ & $0.368 \pm 0.041$ & 0.045 & 0.016 & 0.508 & 0.092 \\
\hline Inferior & $0.378 \pm 0.025$ & $0.387 \pm 0.028$ & $0.360 \pm 0.028$ & 0.001 & 0.222 & 0.025 & $<0.001$ \\
\hline Nasal & $0.384 \pm 0.034$ & $0.390 \pm 0.022$ & $0.385 \pm 0.027$ & 0.619 & & & \\
\hline Average & $0.382 \pm 0.033$ & $0.389 \pm 0.016$ & $0.372 \pm 0.026$ & 0.032 & 0.309 & 0.181 & 0.009 \\
\hline
\end{tabular}

P1: $P$ value for the comparison group between active TAO and inactive TAO $P 2: P$ value for the comparison group between active TAO and normal controls P3: $P$ value for the comparison group between inactive TAO and normal controls

subgroups (all $P<0.05$ ), but exhibited a poor discriminatory power. Figures 2, 3 and 4 showed the detailed ROC curves for different subgroups.

\section{Discussion}

OCTA is a new imaging technology that provides noninvasive fundus angiography, which works without contrast medium and avoids allergies and various contraindications. OCTA relies on the intrinsic motion of the fundus vasculature network to separate stationary structures to identify the blood flow. OCTA can also provide a $3 \mathrm{D}$ partition by comparing $2 \mathrm{D}$ images taken by indocyanine green angiography and fluorescein fundus angiography [18], which avoids artifacts and limitations such as a limited measurement time window and discomfort during inspection. OCTA is reliable, noninvasive, efficient, high quality and safe for fundus vascular imaging $[18,19]$. Fundus perfusion depends on the orbital blood supply, and patients with TAO show orbital perfusion changes caused by pathological changes in orbital tissues. A previous study found certain hemodynamic changes in the ocular vasculature under Doppler imaging, and the condition in the ocular vasculature improved after orbital decompression [9]. Although color Doppler imaging has been widely used in vessel inspection, after certain ocular vasculature changes were detected, fundus perfusion changes can hardly be observed by Doppler imaging. Therefore, OCTA would be a perfect choice for further inspection to evaluate the status in fundus vessels.

In our present study, significant thinner RNFL thickness accompanied by higher IOP level was observed in active TAO patients, and the most affected RNFL quadrants were temporal and inferior quadrants. The superficial retinal vascular plexus may be the capillary network primarily responsible for RNFL nourishment. In our study, the macular superficial vessel density remained unchanged in active TAO patients while the thinning of RNFL was observed, suggesting that losses in the peripapillary RNFL in active TAO patients might have been

Table 4 Associations between OCTA parameters and clinical variables

\begin{tabular}{|c|c|c|c|c|c|c|}
\hline \multicolumn{3}{|c|}{ FAZ area } & \multicolumn{2}{|c|}{ Average vascular density } & \multicolumn{2}{|c|}{ Average perfusion density } \\
\hline & $r$ & $p$ & $r$ & $p$ & $r$ & $p$ \\
\hline Age & 0.136 & 0.224 & -0.114 & 0.308 & -0.076 & 0.500 \\
\hline Proptosis & 0.152 & 0.173 & 0.051 & 0.652 & 0.122 & 0.276 \\
\hline $\mathrm{IOP}$ & 0.274 & $0.013^{*}$ & 0.008 & 0.994 & 0.195 & 0.079 \\
\hline$A L$ & -0.344 & $0.002^{*}$ & 0.118 & 0.292 & 0.025 & 0.821 \\
\hline CCT & 0.093 & 0.406 & -0.010 & 0.927 & -0.057 & 0.610 \\
\hline Average RNFL & -0.070 & 0.531 & 0.169 & 0.129 & 0.113 & 0.312 \\
\hline Average $\mathrm{CT}$ & 0.124 & 0.267 & 0.043 & 0.700 & 0.184 & 0.099 \\
\hline
\end{tabular}

* indicated significant difference with $P<0.05$ 
Table 5 Area under curve with 95\% confidence interval in different subgroups

\begin{tabular}{|c|c|c|c|c|c|c|}
\hline & \multicolumn{2}{|c|}{ Active TAO/inactive TAO } & \multicolumn{2}{|c|}{ Active TAO /Normal } & \multicolumn{2}{|c|}{ Inactive TAO /Normal } \\
\hline & AUC (95\%Cl) & $P$ & AUC (95\%Cl) & $P$ & AUC (95\%Cl) & $P$ \\
\hline RNFL thickness & $0.804(0.704,0.904)$ & $<0.001$ & $0.818(0.701,0.935)$ & $<0.001$ & $0.514(0.368,0.660)$ & 0.849 \\
\hline Choroidal thickness & $0.569(0.424,0.714)$ & 0.353 & $0.814(0.693,0.935)$ & $<0.001$ & $0.828(0.719,0.936)$ & $<0.001$ \\
\hline FAZ area & $0.617(0.468,0.766)$ & 0.117 & $0.711(0.564,0.858)$ & 0.013 & $0.595(0.453,0.737)$ & 0.202 \\
\hline Vascular density & $0.743(0.610,0.877)$ & 0.001 & $0.534(0.371,0.696)$ & 0.692 & $0.682(0.544,0.821)$ & 0.014 \\
\hline Perfusion density & $0.663(0.506,0.820)$ & 0.029 & $0.533(0.367,0.699)$ & 0.699 & $0.699(0.565,0.833)$ & 0.007 \\
\hline
\end{tabular}

influenced by factors other than retinal microcirculation. Active TAO patients often suffer from secondary compressive IOP rise, thus leading to RNFL defects [20, 21]. The enlargement of extraocular muscles and increase of orbital soft tissue volume could cause direct compression of the optic nerve, which also contribute to dysthyroid optic neuropathy. Earlier detection of RNFL thinning would suggest the presence of optic neuropathy, indicating its use in the evaluation of this disease profile.

Significant greater CT was observed in active and inactive TAO eyes as compared to the normal eyes. Çalışkan et al. [6] found the subfoveal CT in active TAO patients was significantly greater than those with inactive TAO or healthy individuals, even after adjusting for age, axial length and IOP. Similar results were observed in another study conducted by Özkan et al. [22]. Besides, $\mathrm{Yu}$ et al. [11] also identified increased CT in TAO patients at different locations in the macular region. Theoretically, one possible explanation for the choroidal variations might be the venous obstruction and congestion, caused by reduce orbital venous drainage, which was the result of increased retrobulbar pressure [22, 23]. Furthermore, the hyperdynamic cardiovascular state of hyperthyroidism could induce an increase in cardiac output, possibly affecting the choroidal perfusion [24].

OCTA has been widely used to analyze the detailed characterization of the retinal and choroidal vasculature in the macular and peripapillary regions [25-27]. Due to the limitations of the analysis software, only superficial vascular plexus in the macular region could be quantitatively analyzed in our study. The FAZ area is a capillaryfree area in the central macula that serves as the most sensitive part of the retina. In our study, the FAZ area was significantly enlarged in active TAO patients. But we didn't detect disintegrity of the vascular arcades surrounding the FAZ area. Previous studies reported that the enlargement of FAZ area more objectively supported the findings of capillary nonperfusion [28, 29]. However, our findings suggested the superficial vascular plexus was increased in inactive TAO patients. The FAZ area measurements showed high reproducibility and repeatability, but the real data vary in different studies. Several possible confounding factors, such as age, sex, spherical equivalent, and axial length, may influence the size of FAZ area [30, 31]. In our study, the FAZ area was positively correlated with IOP $(r=0.274, P=0.013)$, while it was negatively correlated with axial length $(r=-0.344$, $P=0.002)$, which might give a partial explanation.

The macular vascular density and perfusion density of the superficial layer were quantitatively evaluated in $\mathrm{TAO}$ patients. Inactive TAO patients had significantly higher vascular density than that in active TAO and controls. With regard to perfusion density, the pairwise comparison results were not completely consistent. Collectively, the data analysis revealed that inactive TAO patients seemed to have greater perfusion density. In previous studies, Ye at al [32]. reported that active TAO patients presented with an increased retinal microvascular density. Akpolat et al. [33] demonstrated that the temporal and nasal parafoveal vessel density was significantly higher in inactive TAO group, which was consistent with our results. However, Tehrani et al. [34] found active and inactive TAO patients both had significantly lower superficial vessel density in the nasal parafoveal

Table 6 Sensitivity and specificity of different parameters in different subgroups

\begin{tabular}{|c|c|c|c|c|c|c|c|c|c|}
\hline & \multicolumn{3}{|c|}{ active TAO/inactive TAO } & \multicolumn{3}{|c|}{ active TAO/normal } & \multicolumn{3}{|c|}{ inactive TAO/normal } \\
\hline & Cut-off & Sen & Spe & Cut-off & Sen & Spe & Cut-off & Sen & Spe \\
\hline RNFL thickness & 99 & 0.667 & 0.850 & 98 & 0.724 & 0.800 & 103 & 0.485 & 0.621 \\
\hline Choroidal thickness & 258 & 0.545 & 0.700 & 250 & 0.103 & 0.400 & 241 & 0.848 & 0.759 \\
\hline FAZ area & 0.28 & 0.758 & 0.300 & 0.35 & 0.172 & 0.500 & 0.35 & 0.364 & 0.828 \\
\hline Vascular density & 21.6 & 0.697 & 0.800 & 21.6 & 0.345 & 0.800 & 21.6 & 0.697 & 0.655 \\
\hline Perfusion density & 0.381 & 0.697 & 0.700 & 0.381 & 0.483 & 0.700 & 0.376 & 0.848 & 0.517 \\
\hline
\end{tabular}

Sen Sensitivity, Spe Specificity 


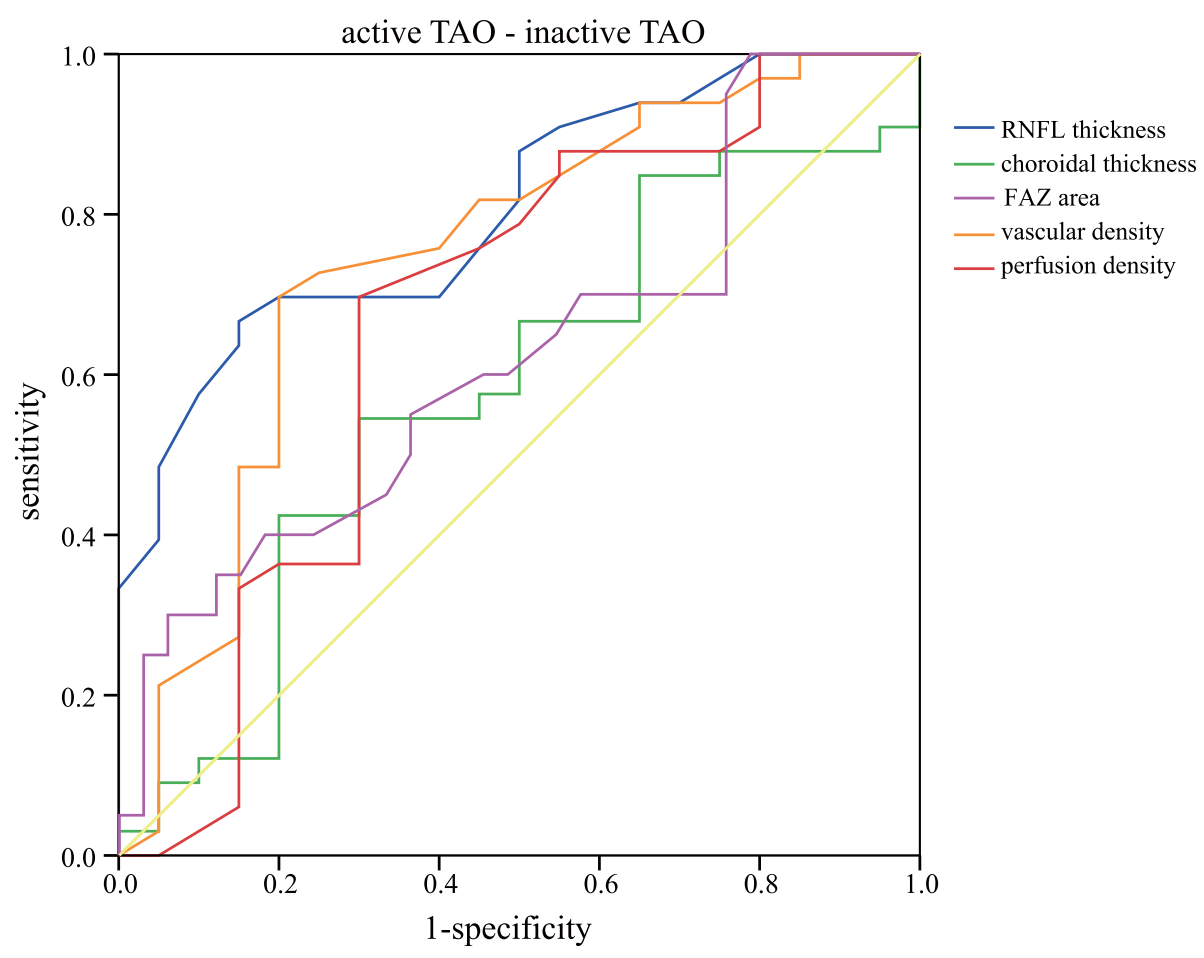

Fig. 2 ROC curves for different parameters to discriminate active TAO from inactive TAO eyes

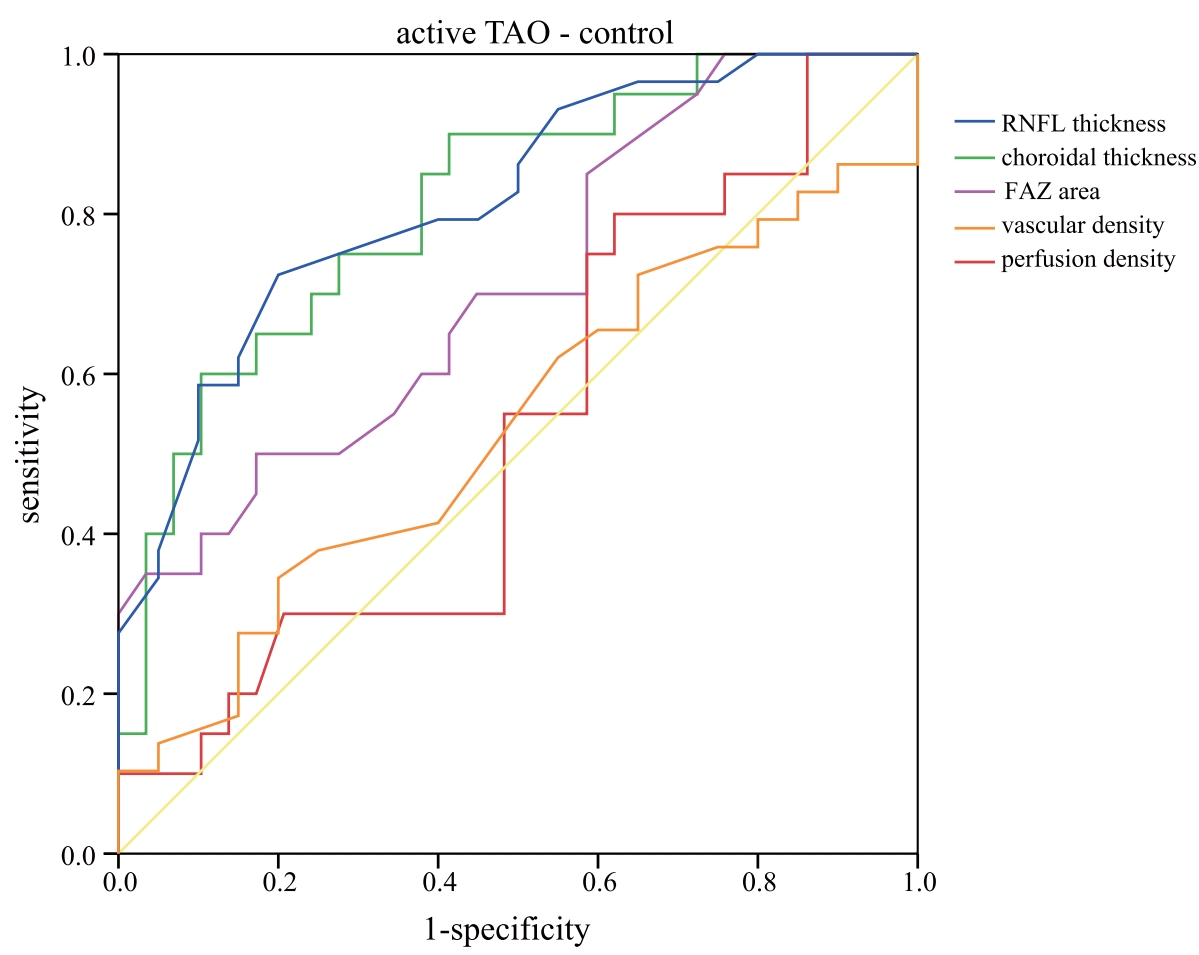

Fig. 3 ROC curves for different parameters to discriminate active TAO from normal eyes 


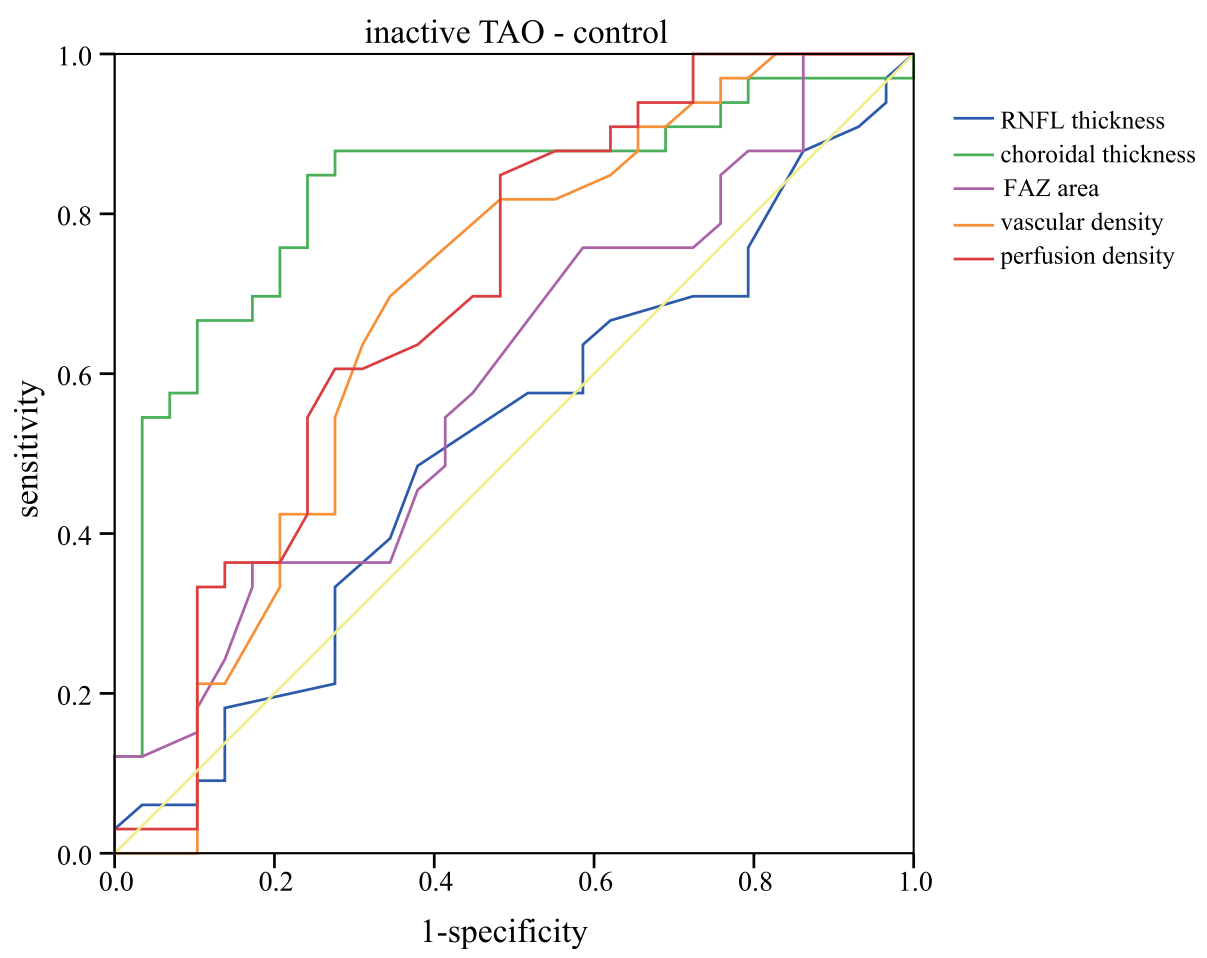

Fig. 4 ROC curves for different parameters to discriminate inactive TAO from normal eyes

sector. Mihailovic et al. [35] also showed inactive TAO patients had decreased vessel density in the superficial OCT angiogram. In these studies, the normal controls were not totally matched to the study population on several nonexperimental factors, such as age, sex, and axial length, which might at least partially explain the discrepancy. Besides, technical differences and variations in patient status, such as inactive or active TAO state or moderate-to-severe status, may also contribute to the incongruity. Further studies with more rigorous design are desiderated to explore the alterations in TAO patients.

These retinal and choroidal changes might be correlated with variations in orbital blood flow. Doppler imaging of orbital vessels revealed that the resistance index (RI) in the ophthalmic artery (OA) was decreased inactive TAO patients, but systolic velocity remained unchanged, suggesting increased blood flow in OA in inactive TAO patients. The RI in the central retinal artery (CRA) was increased, and the velocity and RI in superior ophthalmic vein (SOV) showed no difference compared with that in the control group [9]. The increased blood supply in the ocular vasculature may partly explain the increased vessel density. Walasik-Szemplińska D et al. [9] found that RI in OA decreased in active TAO. Velocity and RI were increased in CRA, while increased RI and decreased velocity were detected in SOV, indicating circulatory disorder in the ocular vasculature. Reverse flow was also observed in SOV, indicating severe stasis in SOV, which usually correlated with enlarged extraocular muscles. The SOV was considered to play important roles in the inflammatory stage in $\mathrm{TAO}$, and recent studies have demonstrated blood flow reduction in the SOV during active TAO, indicating orbital circulation disorder was resulted from a total effect of increasing venous pressure and high RI, which was caused by elevated intraorbital pressure. Autoimmune inflammation in orbital tissues, including interstitial tissues, orbital fat and extraocular muscles, was the main cause of the high intraorbital pressure [3]. Moreover, Onaran et al. [36] observed a reduction in SOV flow among patients after orbital decompression along with the disappearance of the reverse flow. Therefore, we proposed that vascular physiological changes and elevated intraorbital pressure caused by the direct effect of autoimmune inflammation on ocular vessel and orbital tissues lead to variations in fundus blood flow in active TAO, along with effects on RNFL thickness, CT, FAZ, vessel density and perfusion density, as observed in our study.

In the ROC analysis, OCT-derived RNFL thickness and choroidal thickness showed apparent diagnostic ability in TAO, which were consistent with previous studies $[10,18]$. The FAZ area, vascular density and perfusion density also exhibited a significant discriminatory power to distinguish between TAO patients and controls. Ye at al [32]. also showed high diagnostic power to differentiate active TAO patients from normal controls, 
with AUC of 0.97 for superficial retinal density and AUC of 0.8 for deep retinal density. We hypothesize that these parameters may have the predictive value in the diagnosis of TAO. In addition, non-invasive measurements of these parameters are easily accepted by the patients. Clearly, these parameters were poor markers, further investigations are needed to substantiate these findings in a much larger cohort.

As a preliminary study, our present findings have several limitations. First, this was a cross-sectional study without follow-up data, which prevented us to correlate the vascular changes with the disease progression. Second, the morphology of the superficial retinal vessels is not equal to the hemodynamic changes, which may limit our understanding of the pathogenesis. Third, analysis of the retinal vessel parameters was limited to the superficial layer due to the limitation of analysis software, further investigations of deep retinal vessels should be performed to better demonstrating the retinal vessel variations. Fourth, the sample size was relatively small due to the rigorous selection standards. Moreover, the study group included in the current analysis were TAO patients. Dysthyroid patients in the absence of TAO were not enrolled, which may also limit the interpretation of the results to some extent. Nevertheless, our results indicated retinal and choroidal variations in TAO patients, further researches are highlighted to supplement and extend these preliminary results.

\section{Conclusion}

TAO patients had significant variations in RNFL thickness, choroidal thickness, FAZ area and superficial retinal vessels. These parameters appeared to be potential adjuncts in the evaluation of TAO patients.

\section{Abbreviations \\ TAO: Thyroid-associated ophthalmopathy; OCT: Optical coherence tomography; RNFL: Retinal nerve fiber layer; CT: Choroidal thickness; OCTA: Optical coherence tomography angiography; CAS: Clinical activity score; IOP: Intraocular pressure; CCT: Central corneal thickness; AL: Axial length; FAZ: Foveal avascular zone; ROC: Receiver operating characteristic; AUC: Area under curve; RI: Resistance index; OA: Ophthalmic artery; CRA: Central retinal artery; SOV: Superior ophthalmic vein}

\section{Acknowledgements}

We do not have anyone to acknowledge.

\author{
Authors' contributions \\ Conceptualization of the study: ZJL and XS, data acquisition and analysis: \\ LCY, QJ, YC and YJZ, manuscript preparation: LCY and ZJL, revision of \\ manuscript: ZJL and XS. All authors have read and approved the final \\ manuscript.
}

\section{Funding}

Grand from Shanghai Hospital Development Center (SHGC 12016116)

Shanghai Key Laboratory of Visual Impairment and Restoration.

\section{Availability of data and materials}

All data generated or analyzed to support the findings of this study is available in the paper without restriction. The raw data during this study is available from the corresponding author on reasonable request.

\section{Ethics approval and consent to participate}

This research received the ethical approval from Ruijin Hospital, affiliated with Shanghai Jiao Tong University School of Medicine. All procedures were in accordance with the Declaration of Helsinki. All participants signed informed consent forms to participate in the study.

\section{Consent for publication}

Not applicable.

\section{Competing interests}

The authors declare that there were no competing interests regarding the publication of this paper.

Received: 26 April 2020 Accepted: 12 October 2020

Published online: 20 October 2020

\section{References}

1. Bahn RS. Graves' ophthalmopathy. N Engl J Med. 2010;362(8):726-38.

2. Maheshwari R, Weis E. Thyroid associated orbitopathy. Indian J Ophthalmol. 2012;60(2):87-93.

3. Iyer S, Bahn R. Immunopathogenesis of graves' ophthalmopathy: the role of the TSH receptor. Best Pract Res Clin Endocrinol Metab. 2012;26(3):281-9.

4. McAlinden C. An overview of thyroid eye disease. Eye Vis (Lond). 2014;1:1-9.

5. Perri P, Campa C, Costagliola C, Incorvaia C, D'Angelo S, Sebastiani A. Increased retinal blood flow in patients with active graves' ophthalmopathy. Curr Eye Res. 2007;32(11):985-90.

6. Çalışkan S, Acar M, Gurdal C. Choroidal thickness in patients with graves' ophthalmopathy. Curr Eye Res. 2017;42(3):484-90.

7. Sen E, Berker D, Elgin U, Tutuncu Y, Ozturk F, Guler S. Comparison of optic disc topography in the cases with graves' disease and healthy controls. J Glaucoma. 2012;21(9):586-9.

8. Victores AJ, Takashima M. Thyroid eye disease: optic neuropathy and orbital decompression. Int Ophthalmol Clin. 2016;56(1):69-79.

9. Walasik-Szemplińska D, Pauk-Domańska M, Sanocka U, Sudoł-Szopińska I. Doppler imaging of orbital vessels in the assessment of the activity and severity of thyroid-associated orbitopathy. J Ultrason. 2015;15(63):388-97.

10. Sayin O, Yeter V, Ariturk N. Optic disc, macula, and retinal nerve fiber layer measurements obtained by OCT in thyroid-associated ophthalmopathy. J Ophthalmol. 2016;2016:9452687.

11. Yu N, Zhang Y, Kang L, Gao Y, Zhang J, Wu Y. Analysis in choroidal thickness in patients with graves' ophthalmopathy using spectral-domain optical coherence tomography. J Ophthalmol. 2018;2018:3529395.

12. Del Noce C, Vagge A, Nicolò M, Traverso CE. Evaluation of choroidal thickness and choroidal vascular blood flow in patients with thyroidassociated orbitopathy (TAO) using SD-OCT and Angio-OCT. Graefes Arch Clin Exp Ophthalmol. 2020;258(5):1103-7.

13. Durbin MK, An L, Shemonski ND, Soares M, Santos T, Lopes M, et al Quantification of retinal microvascular density in optical coherence tomographic angiography images in diabetic retinopathy. JAMA Ophthalmol. 2017;135(4):370-6.

14. Coscas F, Sellam A, Glacet-Bernard A, Jung C, Goudot M, Miere A, et al. Normative data for vascular density in superficial and deep capillary plexuses of healthy adults assessed by optical coherence tomography angiography. Invest Ophthalmol Vis Sci. 2016;57(9):211-23.

15. Ferrara D, Waheed NK, Duker JS. Investigating the choriocapillaris and choroidal vasculature with new optical coherence tomography technologies. Prog Retin Eye Res. 2016;52:130-55.

16. Bartalena L, Baldeschi L, Dickinson A, et al. Consensus statement of the European group on graves' orbitopathy (EUGOGO) on management of GO. Eur J Endocrinol. 2008;158(3):273-85.

17. Lin Z, Huang S, Huang P, Guo L, Shen X, Zhong Y. The diagnostic use of choroidal thickness analysis and its correlation with visual field indices in glaucoma using spectral domain optical coherence tomography. PLoS One. 2017;12(12):e0189376.

18. Kim AY, Rodger DC, Shahidzadeh A, Chu Z, Koulisis N, Burkemper B, et al. Quantifying retinal microvascular changes in uveitis using spectral-domain 
optical coherence tomography angiography. Am J Ophthalmol. 2016; 171(10):101-12.

19. Koustenis A Jr, Harris A, Gross J, Januleviciene I, Shah A, Siesky B. Optical coherence tomography angiography: an overview of the technology and an assessment of applications for clinical research. Br J Ophthalmol. 2016; 101(1):16-20.

20. Forte R, Bonavolontà P, Vassallo P. Evaluation of retinal nerve fiber layer with optic nerve tracking optical coherence tomography in thyroidassociated orbitopathy. Ophthalmologica. 2010;224(2):116-21.

21. Wei YH, Chi MC, Liao SL. Predictability of visual function and nerve fiber layer thickness by cross-sectional areas of extraocular muscles in graves ophthalmopathy. Am J Ophthalmol. 2011;151(5):901-6.

22. Özkan B, Koçer ÇA, Altintaş Ö, Karabaş L, Acar AZ, Yüksel N. Choroidal changes observed with enhanced depth imaging optical coherence tomography in patients with mild graves orbitopathy. Eye (Lond). 2016; 30(7):917-24.

23. Konuk O, Onaran Z, Ozhan Oktar S, Yucel C, Unal M. Intraocular pressure and superior ophthalmic vein velocity in graves' orbitopathy: relation with clinical features. Graefes Arch Clin Exp Ophthalmol. 2009;247(11):1555-9.

24. Jabbar A, Pingitore A, Pearce SH, Zaman A. Lervasi G, Razvi S. thyroid hormones and cardiovascular disease. Nat Rev Cardiol. 2017;14(1):39-55.

25. Nesper PL, Fawzi AA. Human parafoveal capillary vascular anatomy and connectivity revealed by optical coherence tomography angiography. Invest Ophthalmol Vis Sci. 2018;59(10):3858-67.

26. Borrelli E, Sadda SR, Uji A, Querques G. Pearls and pitfalls of optical coherence tomography angiography imaging: a review. Ophthalmol Therapy. 2019;8(2):215-26.

27. Chua J, Tan B, Ang M, Nongpiur ME, Tan AC, Najjar RP, et al. Future clinical applicability of optical coherence tomography angiography. Clin Exp Optom. 2019;102(3):260-9.

28. Shahlaee A, Pefkianaki M, Hsu J, Ho AC. Measurement of foveal avascular zone dimensions and its reliability in healthy eyes using optical coherence tomography angiography. Am J Ophthalmol. 2016;161(1):50-5.

29. lafe NA, Phasukkijwatana N, Chen X, Sarraf D. Retinal capillary density and foveal avascular zone area are age-dependent: quantitative analysis using optical coherence tomography angiography. Invest Ophthalmol Vis Sci. 2016;57(13):5780-7.

30. Yu J, Jiang C, Wang $X$, Zhu L, Gu PP, Xu H, et al. Macular perfusion in healthy Chinese: an optical coherence tomography angiogram study. Invest Ophthalmol Vis Sci. 2015;56(5):3212-7.

31. Tan CS, Lim LW, Chow VS, Chay IW, Tan S, Cheong KX, et al. Optical coherence tomography angiography evaluation of the parafoveal vasculature and its relationship with ocular factors. Invest Ophthalmol Vis Sci. 2016;57(9):OCT224-34.

32. Ye L, Zhou SS, Yang WL, Bao J, Jiang N, Min YL, et al. Retinal microvasculature alteration in active thyroid-associated ophthalmopathy. Endocr Pract. 2018;24(7):658-67.

33. Akpolat C, Kurt MM, Yılmaz M, Ordulu F, Evliyaoglu F. Analysis of foveal and parafoveal microvascular density and retinal vessel caliber alteration in inactive graves' ophthalmopathy. J Ophthalmol. 2020;2020:7643737.

34. Jamshidian Tehrani M, Mahdizad Z, Kasaei A, Fard MA. Early macular and peripapillary vasculature dropout in active thyroid eye disease. Graefes Arch Clin Exp Ophthalmol. 2019;257(11):2533-40.

35. Mihailovic N, Lahme L, Rosenberger F, et al. Altered retinal perfusion in patients with inactive graves ophthalmopathy using optical coherence tomography angiography. Endocr Pract. 2020;26(3):312-7.

36. Onaran Z, Konuk O, Oktar SÖ, Yücel C, Unal M. Intraocular pressure lowering effect of orbital decompression is related to increased venous outflow in graves orbitopathy. Curr Eye Res. 2014;39(7):666-72.

\section{Publisher's Note}

Springer Nature remains neutral with regard to jurisdictional claims in published maps and institutional affiliations.

\section{Ready to submit your research? Choose BMC and benefit from:}

- fast, convenient online submission

- thorough peer review by experienced researchers in your field

- rapid publication on acceptance

- support for research data, including large and complex data types

- gold Open Access which fosters wider collaboration and increased citations

- maximum visibility for your research: over $100 \mathrm{M}$ website views per year

At BMC, research is always in progress.

Learn more biomedcentral.com/submissions 